(e-migrinter

e-Migrinter

6 | 2010

Rroms \& Gens du Voyage

\title{
Le pentecôtisme : nouveau facteur de mobilité pour les populations tsiganes?
}

Julia Peyron

\section{OpenEdition}

Journals

Édition électronique

URL : https://journals.openedition.org/e-migrinter/1366

DOI : 10.4000/e-migrinter.1366

ISSN : 1961-9685

Éditeur

UMR 7301 - Migrinter

Édition imprimée

Date de publication : 15 octobre 2010

Pagination : 73-82

ISSN : 1961-9685

\section{Référence électronique}

Julia Peyron, «Le pentecôtisme : nouveau facteur de mobilité pour les populations tsiganes ? », eMigrinter [En ligne], 6 | 2010, mis en ligne le 16 janvier 2019, consulté le 20 mai 2021. URL : http:// journals.openedition.org/e-migrinter/1366 ; DOI : https://doi.org/10.4000/e-migrinter.1366 


\section{Le pentecôtisme : nouveau facteur de mobilité pour les populations tsiganes?}

Julia Peyron

$\mathbf{L}$ es motivations au voyage sont de plusieurs ordres au sein des populations tsiganes. Trois de celles-ci interviennent plus significativement, il s'agit des motivations d'ordre familial (descendance, alliances, réseaux, décès, proximité géographique), d'ordre économique (par la nécessité traditionnelle de se déplacer vers la clientèle) et d'ordre religieux. Ces impératifs organisent donc les déplacements. Il apparaît aujourd'hui que les mobilités tsiganes sont

bouleversées, ceci en raison des transformations qu'a connu notre société dans les dernières décennies (urbanisation galopante, complexifications administratives), mais c'est principalement le problème posé par le stationnement qui affecte la mobilité tsigane.
Il faut noter que le voyage proprement dit n'est pas ou plus constitutif de l'identité tsigane, en raison notamment de la très forte sédentarisation. Le clivage nomade/ sédentaire ne correspond plus vraiment à la réalité tant les situations sont diverses et complexes. En effet, chaque famille a des modalités spécifiques d'implantation. Les situations sont très variées entre des familles qui ne voyagent pas du tout, des familles qui voyagent de manière occasionnelle, des familles qui voyagent la moitié de l'année et des familles qui voyagent tout le long de l'année. Pourtant, la notion de voyage revêt une importance symbolique, celle de se définir par rapport aux non-tsiganes, les gadjé.

Traditionnellement les motivations religieuses interviennent lors des pèlerinages catholiques. Or aujourd'hui, avec la diffusion massive du pentecôtisme chez les populations tsiganes en France, de nouvelles mobilités se mettent en place. Dans le contexte actuel, quelle importance prend le facteur de la religion pentecôtiste dans les motivations au voyage?

Ce texte fait état de l'avancement de mes réflexions à partir d'une recherche doctorale en cours. Je présente ici des hypothèses et des questionnements qui nécessitent encore une confrontation avec le terrain.

Un contexte défavorable à la mobilité des populations tsiganes en France

Dans l'Histoire, aucune véritable rébellion tsigane n'est à noter. Les tsiganes sont toujours parvenus à préserver leur identité sans lutte violente. L'identité tsigane n'est donc pas figée, elle est produite en permanence par ceux qui la font vivre, 
autrement dit, par les populations tsiganes elles-mêmes, par les capacités d'adaptation dont elles font preuve. Cependant, aujourd'hui l'équilibre de cette identité paraît menacé. La société française a connu de profonds changements depuis la Seconde Guerre mondiale mais la nature des changements actuels diffère des adaptations précédentes. Les capacités d'adaptation que ces populations ont montré jusqu'à maintenant sont dépassées. Cette menace est principalement le fait des sociétés environnantes qui ont subi d'importantes mutations depuis la Seconde Guerre Mondiale. La France a été affectée dans son peuplement, son économie, son mode de consommation. On assiste à un dépeuplement des régions rurales et à une urbanisation croissante. Ces mutations atteignent aussi les populations tsiganes. Les questions alors soulevées sont d'autant plus fondamentales que les rapports entretenus par les populations tsiganes avec leur environnement conditionnent l'existence de cette minorité. Les contraintes récentes sont autant d'ordre social et économique que réglementaires.

Les Tsiganes sont souvent perçus dans les représentations collectives comme vivant en marge de la société (sous-entendu la société des gadjè). C'est à dire que les tsiganes constituent un groupe qui a sa propre culture, ses valeurs, son histoire particulière, malgré une grande diversité interne. La population tsigane peut être considérée comme marginal au vu des problèmes qu'elle rencontre (accès aux soins, à l'emploi, aux aides sociales, l'éducation, l'accès aux devoirs citoyens, la précarité, l'exclusion). Les tsiganes sont victimes de fortes discriminations liées essentiellement à leur style de vie (mode de vie communautaire, mode de vie caractérisé par le nomadisme même si aujourd'hui la plupart sont sédentaires). Cependant, on peut dire également que les tsiganes sont aussi laissés à la marge par la société : par exemple, les aires d'accueil sont souvent installées aux limites des communes.
Mais, si les tsiganes vivent parfois en marge de la société, ils ne vivent cependant pas aux marges de la société (Humeau, 1995). Ils vivent au cœur même de cette société. Les tsiganes sont traditionnellement tout à fait intégrés dans la société, de par leur économie, leur circulation et l'utilisation du territoire (finalement on n'est pas surpris de voir des caravanes), la scolarisation des enfants... Ils ne vivent en aucune façon de manière indépendante et autonome. Mais les Tsiganes ont un rôle d'éternels étrangers, la population les voit fréquemment comme des immigrés. De nombreux auteurs parlent de nomadisme parasitaire (Liégeois, 1983) dans le sens où les tsiganes ne sont pas des nomades qui vivent en autarcie, mais des nomades qui se sont adaptés pour vivre en symbiose avec la population sédentaire. Par ce nomadisme parasitaire, on entend un nomadisme qui s'est développé aux dépens de la société des gadjé.

Les bouleversements sociaux actuels concernent surtout le mode de vie des tsiganes. Les tsiganes, au même titre que le reste de la population, se trouvent intégrés dans de nouveaux modes de consommation. Ainsi ils modifient leurs façons de vivre. Cette situation éloigne les familles des pratiques traditionnelles qui ne correspondent plus à la réalité des jeunes. Ce sont ces derniers les plus touchés par ces évolutions.

Il est également important d'intégrer dans les bouleversements sociaux la sédentarisation, conséquence de l'urbanisation. Même si le clivage nomadisme/sédentarisation est assez complexe et relatif à chaque famille, de moins en moins de familles conservent un mode de vie centré sur le voyage. L'urbanisation croissante modifie les caractéristiques de la mobilité. Les tsiganes suivent le mouvement général d'urbanisation de la population française. La densification du bâti a pour conséquence majeure la destruction de lieux de halte (entretien avec $\mathrm{Mr}$ Cuille, viceprésident du Comité de Coordination pour 
la Promotion et la Solidarité avec les migrants et les tsiganes, 11/02/2008). D'autre part les caravanes sont de plus en plus visibles dans le paysage urbain, et dérangent par conséquent davantage. Une ville est constituée de zones habitables, de zones de travail et de zones de circulation. Mais les tsiganes ne sont acceptés dans aucun de ces espaces.

Ces bouleversements sont fondamentaux dans le sens où le facteur économique est un moteur premier de la mobilité tsigane. Ainsi ces bouleversements affectent donc la circulation des populations tsiganes. L'économie tsigane est fortement imbriquée aux sociétés d'accueil. Cette économie tsigane s'insère dans l'économie de la société majoritaire, qui en fixe le plus souvent les conditions même d'existence. Cette économie fonctionne sur un réseau de connaissances important entre tsiganes et non-tsiganes. Ces réseaux permettent l'appropriation d'un secteur: c'est parce qu'ils sont connus qu'ils vont revenir, grâce au fonctionnement du bouche à oreille entre «clients». Le fonctionnement de cette économie est souvent ignoré, négligé, voir méprisé. Ce mépris semble dû à une invisibilité et à une méconnaissance de cette économie, nourrissant les stéréotypes les plus répandus.

Concrètement, qu'est ce que l'économie traditionnelle tsigane? Un tsigane a plusieurs compétences, combines, et plusieurs métiers. Cette polyvalence lui permet de s'insérer plus facilement dans l'endroit où il se trouve. Les métiers traditionnels les plus répandus sont la chine, la récupération de métaux et de ferraille, le rempaillage, la vente sur les marchés, les stands de foire, les fêtes foraines, l'étamage, l'aiguisage, ou encore les activités du cirque et du spectacle. Chaque groupe familial est plus ou moins spécialisé dans certains domaines. Les tsiganes interviennent dans des secteurs où les non-tsiganes ne sont pas compétitifs ou intéressés. On voit bien que les activités économiques sont essentiellement des prestations fournies à des non-tsiganes (fournitures intermittentes de marchandises, main d'œuvre, services).

L'itinérance est un élément indispensable à l'exercice des métiers traditionnels. Le nomadisme tsigane est un nomadisme dit commercial. Les tsiganes ont mis en place une territorialisation économique, ce qui signifie que leurs déplacements sont planifiés et qu'ils sont liés à des impératifs économiques (nécessité de se déplacer vers la clientèle), sociaux et familiaux. Tout cela forme un réseau. L'organisation du voyage est réfléchie et cohérente. Aujourd'hui le voyage se fait de moins en moins en famille, et de plus en plus souvent de manière individuelle et à la journée (cela peut s'expliquer par le phénomène de la sédentarisation du, entre autres, à la scolarisation des enfants ou la difficulté de trouver des places en aires d'accueil). Le voyage est de plus en plus difficilement conciliable avec la société moderne.

Toutefois, il faut aujourd'hui repenser l'économie tsigane à la lumière des grands bouleversements dont est victime la société tsigane. On a assisté dans les dernières décennies à une disparition des métiers traditionnels qui ne trouvent plus de place dans les sociétés actuelles et à un développement de nouvelles activités économiques. Ainsi, la mécanisation des travaux de cueillette, l'apparition des déchetteries, l'organisation de la récupération des métaux, les marchés saturés, réduisent les possibilités de travail des tsiganes. De plus, les politiques visant à une meilleure visibilité des activités tsiganes vont à l'encontre de leur fonctionnement économique qui se situe en marge et dans les failles juridiques du système dominant. Les réglementations se complexifient fortement, comme la législation relative au travail indépendant et à la protection des consommateurs. Par exemple pour le porte à porte : les clients ont maintenant $48 \mathrm{~h}$ pour se rétracter. Malgré des compétences 
importantes, des savoir-faire réels, les tsiganes n'ont pas l'expérience salariée ou le niveau scolaire requis pour obtenir des diplômes, aujourd'hui indispensables pour mener une activité indépendante.

On assiste à une cassure entre les tsiganes qui ont su s'adapter aux contraintes, utiliser les services juridiques et comptables et accepter une relative spécialisation et ceux qui sont engagés dans un processus de paupérisation et pour lesquels les revenus des aides sociales sont supérieurs aux revenus de l'activité professionnelle. En effet de nos jours, de nombreux tsiganes perçoivent des aides sociales et en vivent. Ces aides constituent des revenus fixes et sûrs, mais témoignent d'un phénomène de désinsertion socioprofessionnelle, la reconversion des métiers traditionnels ne se passant pas toujours dans de bonnes conditions. Cela signifie que les tsiganes se sentent dépassés par le monde économique et professionnel actuel.

Des bouleversements réglementaires entraînent des difficultés à stationner toujours plus importantes, même si la liberté d'aller et venir est la règle et constitue un principe général pour les citoyens de l'Europe qui peuvent alors circuler librement sur le territoire et peuvent également le quitter. Ce principe est rendu relatif par un certain nombre de cas particuliers qui posent, pour les autorités, le problème de concilier cette liberté avec le maintien de l'ordre public. De plus, quelle est la réalité de cette liberté d'aller et venir si elle n'est pas accompagnée de la liberté de s'arrêter? En effet, cette liberté reste conditionnée par la décision des pouvoirs de police du maire sur le territoire de sa commune. Aucun texte ne proclame que le stationnement est une liberté publique.

La planification urbaine et la décentralisation territoriale aboutissent à une organisation planifiée de l'espace. Les schémas départementaux se multiplient ainsi que les plans d'occupation des sols. Ils réduisent considérablement les possibilités de stationnement en évitant l'inscription de lieux de séjours dans ces planifications. Les agglomérations ont pour objectif d'attirer toujours plus d'habitants. Aussi les espaces se comblent, les contraintes se font plus pressantes. Les terrains libres sont la cible de projets urbanistiques. Les espaces se ferment et tout lieu vaquant est investi.

Ainsi, il s'avère que la liberté de stationner est de plus en plus réduite par des interdictions et des réglementations. Si on ne parle pas directement du stationnement des populations tsiganes, il est question du stationnement des caravanes. Le code de l'urbanisme réglemente de manière très précise le stationnement des caravanes, l'occupation du sol dans le respect des règles d'urbanisme, de l'environnement et de la sécurité publique. En l'absence de terrains de passage et de séjour, il ne peut y avoir une interdiction totale et absolue de stationnement.

Ainsi, les tsiganes qui construisaient leur identité sur la mobilité sont de plus en plus contraints de se sédentariser face aux mutations de la société française. Toutefois, la religion pentecôtiste semble leur redonner de nouveaux motifs pour reprendre la route.

\section{La religion pentecôtiste, un nouvel essor}

Le pentecôtisme est une branche du protestantisme. Il se différencie des autres mouvements chrétiens et protestants par la croyance dans les dons spirituels du SaintEsprit manifesté au $1^{\circ}$ siècle par les apôtres rassemblés lors du jour de la Pentecôte (le don le plus reconnu est le "parler en langues » dit aussi glossolalie ou encore le don de guérison par la prière). Le pentecôtiste est une religion qui se montre dans l'air de son temps. Elle évolue dans la société et s'inscrit dans un contexte. Ainsi elle n'est pas autonome de la culture et de l'histoire des populations concernées. 
Actuellement se développe un phénomène pentecôtiste très fort chez les tsiganes. Les premiers baptêmes datent des années 1950. Le premier rassemblement tsigane a lieu à Brest en 1956. Très vite on assiste à une explosion des rassemblements. Aujourd'hui, ce mouvement est tout à fait actuel et fondamental. La montée en puissance et l'importance du mouvement pentecôtiste est un trait essentiel à prendre en compte dans l'évolution en cours du monde tsigane.

Pour comprendre l'ampleur que cette religion a prise chez les tsiganes, il faut se pencher sur le discours qu'elle produit. Le pentecôtisme est basé sur le message d'amour du Christ. Ce dernier a une signification particulière pour les tsiganes en tant qu'exclus. On a souvent appelé le pentecôtisme, dans d'autres situations, la religion des persécutés ou la religion des pauvres (Fer, 2005). Cette religion est active particulièrement auprès des populations minoritaires. Auprès des tsiganes, une telle adhésion peut témoigner de la réalité d'une certaine crise identitaire. Le pentecôtisme serait-il un moyen de lutter contre l'emprise de la société environnante? Il faut alors replacer ce phénomène par rapport au contexte difficile que connaissent les populations tsiganes, notamment les stéréotypes dont ils font l'objet, ou les différentes lois discriminantes auxquelles ils sont ou ont été soumis. Le discours pentecôtiste affirme la transformation du converti en un homme nouveau. On entend parler de «bons tsiganes », comme s'il y avait des mauvais tsiganes desquels il était important de se démarquer. Le pentecôtisme est compris comme donnant une visibilité sociale positive.

La religion pentecôtiste connaît donc un engouement spectaculaire chez les tsiganes, autant au niveau de la démonstration de la foi, qu'au niveau du nombre de convertis. En quelques décennies, cette religion s'est imposée chez un nombre considérable de tsiganes, et continue à se répandre dans cette communauté. Quantifier le nombre de fidèles est une opération encore délicate. De plus, on observe une grande diversité dans le vécu, la pratique de la foi selon les régions, et selon les familles pentecôtistes. Toutefois, ce sentiment religieux peut être très fort, voire adopter des aspects sectaires. Certaines personnes en arrivent jusqu'au reniement total de leur identité face à la religion. Celleci devient alors la priorité absolue.

Le pentecôtisme qui touche les tsiganes de tous les groupes, nomades aussi bien que sédentaires se vit partout en France dans des lieux de culte fixes (des bâtiments quelconques, non divin) et dans des lieux de culte itinérants, que l'on appelle «conventions ». Les grands rassemblements que sont les missions (qui réunissent quelques familles tsiganes) et les conventions (qui réunissent plusieurs centaines, voire milliers de familles) sont des moments forts $\mathrm{du}$ vécu de la religion. La religion pentecôtiste est donc organisée autour de lieux et de temps de rassemblement. Ces rassemblements sont de tailles diverses selon qu'il s'agit de rassemblements locaux, régionaux, nationaux, ou internationaux. Ces conventions ont principalement lieu entre le mois de mars et d'octobre. L'été tout le territoire de la France est alors parsemé de ces rassemblements. Les missions sont plutôt locales, alors que les conventions, plus importantes peuvent être régionales, nationales, voire internationales. La prise en compte de la distribution régionale est importante. Ces conventions se multiplient sur le territoire français. Celles-ci sont généralement mixtes, mais ils existent aussi des conventions propres à chaque groupe, mais qui restent ouvertes à chacun.

En termes d'organisation, les grands rassemblements sont étonnants. Le pentecôtisme déplace des foules impressionnantes. Les tsiganes ont acquis les capacités d'organiser leurs conventions, qui rassemblent parfois plus de 2000 caravanes, d'avoir des relations avec les autorités 
municipales, préfectorales, de sécurité. En effet, les pasteurs sont formés aujourd'hui à organiser ces rassemblements, et à négocier avec les autorités locales. Ils ont aussi une organisation interne remarquable. Lors de ces conventions règne une grande discipline. Ces conventions se composent de séances de prédication, de chants, de prières, de témoignages de foi et de quête, elles sont très ouvertes, invitent et accueillent très facilement des personnes extérieures.

Outre l'intérêt religieux proprement dit, ces manifestations ont plusieurs intérêts pour les familles tsiganes qui y participent. Dans un premier temps, elles favorisent la concentration occasionnelle de la parenté, des retrouvailles et permettent la rencontre entre jeunes gens. Par ailleurs, elles représentent un marché économique important. En effet leur durée étant de quelques jours à 2-3 semaines, les familles doivent continuer à travailler durant cette période. Ces rassemblements sont alors de fabuleux marchés, des lieux de rencontres, d'échanges, d'entente, d'accords. Les conventions sont marquées par un coté très mercantile. Ainsi de nouvelles dynamiques économiques peuvent émerger, avec de nouveaux circuits, la rencontre de nouvelles personnes.

L'organisation des conventions se fait autour de la rencontre du temps sacré et du temps profane. Les cultes ont lieu principalement le matin et le soir, entre temps les familles vaquent à leurs occupations quotidiennes. Les familles arrivent généralement avant la convention proprement dite et prospectent les alentours. Puis c'est le temps de la mise en place du lieu. Enfin, les familles se dispersent rapidement après la convention (Loizeau, 2000).

Ce phénomène est une source de remobilisation autour $\mathrm{du}$ voyage. Les conventions deviennent un moyen de reprendre le voyage, notamment pour des familles ayant arrêté par crainte des représailles, et pour celles qui n'avaient pas assez de moyens pour affronter les difficultés. En effet, faire partir jusqu'à mille caravanes est très difficile. Par des négociations entre les pasteurs et les autorités, les tsiganes peuvent en général stationner sans trop de problèmes. De nombreuses familles ont compris que suivre les conventions permettait de limiter de manière considérable les expulsions. Ce serait alors une solution tout à fait adaptée que les tsiganes ont trouvé pour stationner sans problème durant une à deux semaines. D'autant plus que l'on retrouve ces conventions partout en France. Il est important de préciser toutefois, que si les conventions sont un " alibi à la mobilité ${ }^{1}$ » et sont ouvertes à tous, ce système profite surtout aux familles qui ont des revenus suffisants pour prendre la route. Ainsi, la pratique de la mobilité serait-elle liée à une quelconque idée d'ascension sociale.

\section{Le stationnement : lois sur les grands rassemblements}

Ces rassemblements amènent à prendre en compte certaines notions corollaires telles la scolarisation, la santé ou le logement. Il est important d'analyser les modalités d'accueil et de stationnement pour ces familles en déplacement lors des conventions. En effet, la régularité des grands rassemblements pentecôtistes, particulièrement en période estivale, appelle des réponses spécifiques en termes de lieux d'accueil. L'importance de ces rassemblements ne permet pas aux familles d'utiliser les aires d'accueil de taille trop modeste.

Pour travailler cette question de l'accueil des rassemblements il faut se pencher sur la loi Besson, loi qui impose une

\footnotetext{
1 Céline Bergeon: Rroms et Voyageurs : pratiques circulatoires et attaches territoriales au miroir des politiques publiques de stationnement et de circulation: l'exemple des Rroms du Poitou-Charentes (France) et de la région Wallonne (Belgique). Thèse de Doctorat en cours.
} 
visibilité des populations tsiganes. Une première loi Besson du 31 mai 1990 vise alors à mettre en œuvre un droit au logement pour tous. Dans cet objectif, l'article 28 de la loi du 31 mai 1990 est consacré aux gens du voyage. Il précise que toute commune de plus de 5000 habitants prévoit les conditions de passage et de séjour des gens du voyage sur son territoire, par la réservation de terrains aménagés à cet effet. Dès la réalisation de l'aire d'accueil définie à l'alinéa ci-dessus, le maire ou les maires des communes qui se sont groupés pour la réalisation pourront, par arrêté, interdire le stationnement des gens du voyage sur le reste $d u$ territoire communal. Devant la quasi-absence d'application de cette loi (notamment à cause du manque d'échéances et de sanctions), la seconde loi Besson, loi du 5 Juillet 2000 porte spécifiquement sur l'accueil et l'habitat des "gens du voyage», population alors présentée, sans aucune référence à un niveau de vie comme population défavorisée. Elle modifie le dispositif prévu dans l'article 28 de la première loi Besson du 31 mai 1990. Le double objectif avoué de cette nouvelle loi Besson est d'une part de répondre à l'aspiration des " gens du voyage » à pouvoir stationner dans des conditions satisfaisantes, et d'autre part d'offrir aux élus locaux des moyens à l'encontre des installations illicites.

Le schéma départemental est l'outil mis en place pour décrire le dispositif d'accueil des "gens du voyage », il en est en quelque sorte le pivot. Il détermine entre autres les communes concernées. Ce document est le bilan de l'analyse des besoins par départements. La création de la commission consultative des «gens du voyage» permet le suivi du schéma départemental et sa mise en œuvre. La commission est composée de représentants de l'Etat, du Conseil Général, de la CAF, d'élus, et d'associations. Le schéma est révisé tous les 6 ans, prévoit le maillage du territoire en aires d'accueil et la détermination des emplacements désignés pour les grands rassemblements.
Ce qui nous intéresse dans le cadre de cette étude est la question des aires de grands passages et les aires de grands rassemblements, aires inscrites également dans les schémas départementaux. Les aires de grands passages se définissent comme des terrains visant à accueillir de façon discontinue, sur de courtes durées (moins de 15 jours), et à des périodes identifiées de grands groupes (de 50 à 200 caravanes), qui viennent de façon organisée sur des lieux de passage connus et récurrents. Ces aires sont utilisées comme étapes par des clans familiaux larges sur la route des rassemblements religieux, rassemblant un nombre bien supérieur de caravanes. Quant aux aires de grands rassemblements, elles se doivent d'être beaucoup plus vastes.

L'article $1^{\text {er }}$-II-alinéa 3 de la loi du 5 Juillet 2000 stipule que le schéma départemental détermine les emplacements susceptibles d'être occupés temporairement à l'occasion de rassemblements traditionnels ou occasionnels et définit les conditions dans lesquelles l'Etat intervient pour assurer le bon déroulement de ces rassemblements. Les aires de grands passages sont en principe d'une capacité suffisante pour 200 caravanes. Ces aires ne peuvent être utilisées par les populations tsiganes que sur de courtes périodes de quelques jours à quelques semaines. Ces aires ne nécessitent pas d'aménagement ou de construction justifiant un permis de construire. Elles peuvent donc être situées hors des zones urbanisées et constructibles des plans locaux d'urbanisme. Il peut par exemple s'agir d'espaces naturels (champs, prés). Les familles tsiganes interrogées à Toulouse évoquent l'envie d'un terrain le moins bétonné possible, ombragé et suffisamment vaste pour pouvoir disposer les caravanes à leur convenance et y installer un chapiteau. Ces familles soulignent aussi l'importance de désigner un terrain proche de commerces.

Les communes prennent en charge la mise en place des équipements ponctuels minimums : une arrivée d'eau et d'électricité, des sanitaires, un ramassage d'ordure. Quant 
à l'Etat, il s'engage à remplir le rôle de coordonnateur. Il transmet l'analyse des besoins aux communes qui se chargent à leur tour de transmettre au préfet des propositions d'emplacements. Ceci se fait d'après les pasteurs évangéliques tsiganes qui acceptent d'envoyer leur circuit annuel et les dates de séjour proposées dans chaque département concernés.

Deux circulaires viennent compléter la loi Besson. La première est la circulaire 2001-49 du 5 Juillet 2001. Elle stipule que compte tenu du développement, constaté au plan national, de groupes importants voyageant ensemble, les départements devront disposer de capacités d'accueil adaptées aux besoins de ces groupes, qui incluent les groupes convergeant vers les lieux de grands rassemblements traditionnels ou occasionnels, quelques temps avant ou après ces rassemblements eux-mêmes. Il est donc important de distinguer les "grands passages» qui ne dépassent pas généralement les 200 caravanes et qui ne sont connus que deux ou trois mois avant leur passage, des "rassemblements traditionnels» ou occasionnels qui sont, eux, connus longtemps à l'avance et regroupent un nombre bien supérieur de caravanes.

La seconde est la circulaire du 8 juillet 2003. Elle prend en compte les courtes haltes de groupes de 50 à 200 caravanes qui convergent vers les lieux de grands rassemblements. Cette circulaire demande aux communes d'organiser l'accueil de ces groupes afin de réunir les meilleures conditions de sécurité. Ainsi les terrains mis à disposition sont à rechercher dans le patrimoine de l'Etat.

\section{Un développement au détriment de la religion catholique? \\ Les conventions pentecôtistes face aux pèlerinages catholiques}

Les Tsiganes en France ont toujours été jusqu'à cette dernière décennie majoritairement catholiques. Pour comprendre le phénomène pentecôtiste, il est nécessaire de comprendre l'implication des tsiganes au sein de l'église catholique jusqu'à l'émergence du mouvement pentecôtiste. A l'heure actuelle il existe une sérieuse concurrence entre le mouvement catholique et le mouvement pentecôtisme. Ces divisions vont même jusqu'à atteindre les unités familiales.

Pourquoi certaines familles adhérentelles davantage à religion pentecôtiste qu'à la religion catholique ? La religion catholique a tendance à marginaliser les tsiganes. Souvent l'authenticité de leur foi est mise en doute (pas de mariage religieux, manque d'assiduité aux cultes dominicaux...). Ainsi, il y a peu de reconnaissance dans le catholicisme, où les tsiganes sont considérés comme de mauvais pratiquants. Avec le pentecôtisme les tsiganes ont enfin l'impression d'avoir une religion qui leur appartient, notamment avec l'existence de la MET (Mission Évangélique Tsigane).

$\mathrm{La}$ figure $\mathrm{du}$ pasteur dans le pentecôtisme joue un rôle important et prosélyte. Le pasteur est chargé de gérer administrativement le mouvement pentecôtiste, c'est-à-dire qu'il organise le mouvement mais n'a pas de caractère sacré. Le mouvement pentecôtiste tsigane forme ses propres pasteurs. La formation est légère et adaptée. Les pasteurs sont de plus en plus nombreux, certains apprennent à lire et à écrire dans le but de pouvoir administrer le culte. Les pasteurs rentrent dans un autre type de relation avec l'étranger, qui n'est pas un rapport d'ennemi, ainsi ils sont plus écoutés des tsiganes. Aussi, les pouvoirs publics s'intéressent-ils à ce mouvement. La figure du pasteur est importante, d'une part parce que c'est lui qui va gérer les conventions (rassemblements), les déplacements, c'est aussi lui qui va permettre la cohésion des familles autour du mouvement.

La religion pentecôtiste connaît donc un engouement spectaculaire chez les populations tsiganes. Ce phénomène est une 
source de remobilisation autour du voyage. C'est une solution adaptée que les tsiganes ont trouvé pour stationner sans craindre l'expulsion pour une ou deux semaines. D'autant plus que l'on retrouve ces conventions un peu partout en France, voire en Europe.

Face à cette organisation du voyage autour des conventions, les pèlerinages catholiques sont encore toujours fortement suivis. Ce sont des moments aussi importants qu'une communion d'Église. Les deux pèlerinages les plus importants sont les Saintes-Maries-de-la-mer (fréquenté surtout par les gitans) et Lourdes (fréquenté par les gitans et les manouches). Ces pèlerinages sont très médiatisés. Les Saintes-Maries-dela-mer sont la manifestation d'une réalité culturelle et ethnique, mais ce pèlerinage paraît très folklorique. Celui-ci est caractérisé par une ferveur considérable de la part des Tsiganes qui s'y rendent. Ce pèlerinage conforte l'image des Tsiganes comme marginaux de l'église catholique, ils apparaissent comme une minorité. Ils font leur propre procession, comme s'ils étaient exclus des processions des gadjé. Ce serait une façon différente de s'approprier le lieu sacré, une façon différente d'exprimer sa foi (Loizeau, 2000).

L'élément important et surprenant est que certaines familles suivent à la fois les conventions pentecôtistes et les pèlerinages catholiques. Des catholiques qui s'insèrent dans les conventions et des pentecôtistes qui se déplacent pour les pèlerinages. Ce qui fait dire au Dr Guiraud, président du CCPS (Comité de Coordination pour la Promotion et la Solidarité des migrants et des tsiganes) que les Tsiganes sont très acuméniques. En effet avec les Saintes-Maries en mai, Lourdes fin août, et les conventions le reste de l'été, les Tsiganes peuvent alors organiser leur voyage autour de rencontres religieuses presque la moitié de l'année.

\section{Conclusion}

Avant d'atteindre la convention «finale» qui aura alors sa taille maximale, quelques familles se réunissent, se rassemblent, se fixent souvent ensemble avec pour objectif d'atteindre le lieu où l'événement pentecôtiste se tiendra. Ces parcours sont l'occasion entre autre de propager la "parole de Dieu». Les rassemblements participent à la structuration des espaces parcourus des familles tsiganes. Ils participent à l'insertion géographique de chaque groupe familial selon un réseau particulier. L'élaboration de l'espace parcouru des tsiganes se fait par la prise en compte de plusieurs facteurs.

L'espace parcouru des tsiganes reflète «leur polygone de vie» (Humeau, 1995) qui désigne l'ensemble des lieux constituants les bases géographiques de l'espace parcouru. Chaque famille a cependant des modalités spécifiques d'implantation, selon l'ancienneté de la relation au territoire, des stratégies familiales ou encore des dynamiques personnelles. Les processus spatiaux sont alors variés.

Enfin, la diversité du mouvement demeure un caractère majeur. En effet, selon les groupes, les familles, les individus, les régions, l'histoire des lieux, le mouvement revêt des réalités diverses et parfois même de fortes dissensions internes. Dans un contexte de redéfinition des relations des populations tsiganes avec la société environnante, la religion pentecôtiste est à prendre en compte, d'autant plus que cette dynamique évolue très vite. Ce mouvement me semble être une clé pour comprendre l'évolution du monde tsigane.

À travers le pentecôtisme, la mobilité tsigane a évolué et semble avoir (re)pris de l'ampleur, mais dans quelle mesure? Sous 
quelle forme cette religion est-elle utilisée par les Tsiganes? Quelle configuration géographique prend cette insertion spatiale?

Julia Peyron Doctorante en géographie Laboratoire EEE (Europe, Européanité, Européanisation)

Université Bordeaux III juliapeyron@hotmail.com

\section{Bibliographie}

Bodigoni, Marc (2004) Sara aux Saintes Maries de la Mer. Métaphore de la présence gitane dans le monde des gadjé, Etudes Tsiganes, $\mathrm{n}^{\circ} 20$, pp.12-35.

Charlemagne, Jacqueline (1993) Où en est le droit des minorités? L'exemple Tzigane, Etudes Tsiganes, vol. 2, pp. 8-31.

Fer, Yannick (2005) Le pentecôtisme en Polynésie Française. L'évangile relationnel, Genève, Labor et Fides, 500 p.

Humeau, Jean-Baptiste (1995) Tsiganes en France. De l'Assignation au droit d'babiter, Paris, L'Harmattan, 409 p.

Le Cossec, Clément (1985) Phénomène pentecôtisme ou réveil religieux?, Etudes Tsiganes, $\mathrm{n}^{\circ} 1 / 85$, pp. 19-21.

Liégeois, Jean-Pierre (1983) Tsiganes, Paris, Maspero, 371 p. (Petite Collection Maspero, $\left.\mathrm{n}^{\circ} 276\right)$.

Loizeau, Gaëlla (2000) "Tsiganes"et "Chrétiens" entre autres. Étude des processus de l'ethnicité tsigane à travers le phénomène pentecôtiste, Bordeaux, Université de Bordeaux III, 175 p. Mem. Maitrise : Ethnol. : Bordeaux : 2000.

Rothéa, Xavier (2003) France, Pays des droits des Roms, Lyon, Carobella ex-natura, 120 p.

Williams, Patrick (dir.) (1989), Tsiganes: Identité, Evolution, Paris, Syros Alternatives, $534 \mathrm{p}$. 